\title{
Synthesis and Characterization of ( $\left.{ }^{\mathrm{DIPP}} \mathrm{CCC}\right) \mathrm{Fe}$ Complexes: A Zwitterionic Metalation Method and $\mathrm{CO}_{2}$ Reactivity
}

\author{
Bailey J. Jackson, Daniel C. Najera, Ellen M. Matson, Toby J. Woods, \\ Jeffery A. Bertke, and Alison R. Fout*
}

School of Chemical Sciences, University of Illinois at Urbana-Champaign, 600 S. Matthews Ave. Urbana, IL 61801.

NMR and IR spectra of $\mathrm{H}_{2}\left[{ }^{\mathrm{DIPP}} \mathrm{CCC}\right] \mathrm{Fe}(\mathrm{II}) \mathrm{Cl}_{3}(\mathbf{1})$

S2

NMR spectra of ( $\left.{ }^{\mathrm{DIPP}} \mathrm{CCC}\right) \mathrm{Fe}(\mathrm{II}) \mathrm{H}\left(\mathrm{PMe}_{3}\right)\left(\mathrm{N}_{2}\right)\left(\mathbf{2}-\mathrm{PMe}_{3}\right)$

S3

NMR spectra of $\left({ }^{\text {DIPP }} \mathrm{CCC}\right) \mathrm{Fe}(\mathrm{II}) \mathrm{H}\left(\mathrm{PPh}_{3}\right)\left(\mathrm{N}_{2}\right)\left(\mathbf{2}-\mathbf{P P h}_{3}\right)$

S4

NMR spectra of ( $\left.{ }^{\mathrm{DIPP}} \mathrm{CCC}\right) \mathrm{Fe}(\mathrm{II}) \mathrm{H}(\mathrm{Py})\left(\mathrm{N}_{2}\right)$ (2-Py)

S5

NMR spectra of ( $\left.{ }^{\mathrm{DIPP}} \mathrm{CCC}\right) \mathrm{Fe}(\mathrm{II}) \mathrm{H}(\mathrm{CO})_{2}$ (4-CO)

S6

NMR spectra of ( $\left.{ }^{\mathrm{DIPP}} \mathrm{CCC}\right) \mathrm{Fe}(\mathrm{II}) \mathrm{H}\left({ }^{\mathrm{t}} \mathrm{BuNC}\right)_{2}$ (4-'BuNC)

S7

NMR spectra of ( $\left.{ }^{\mathrm{DIPP}} \mathrm{CCC}\right) \mathrm{Fe}(\mathrm{II}) \mathrm{Cl}\left(\mathrm{PMe}_{3}\right)_{2}$ (5)

S8

NMR and IR spectra of ( $\left.{ }^{\mathrm{DIPP}} \mathrm{CCC}\right) \mathrm{Fe}(\mathrm{III}) \mathrm{Cl}_{2}\left(\mathrm{PMe}_{3}\right)(\mathbf{6 a})$

S9

NMR and IR spectra of ( $\left.{ }^{\mathrm{DIPP}} \mathrm{CCC}\right) \mathrm{Fe}(\mathrm{III}) \mathrm{Cl}\left(\mathrm{PMe}_{3}\right)_{2}\left(\mathrm{PF}_{6}\right)(\mathbf{6 b})$

$\mathrm{S} 10$

NMR and IR spectra of ( $\left.{ }^{\mathrm{DIPP}} \mathrm{CCC}\right) \mathrm{Fe}(\mathrm{II})\left(\kappa^{2}-\mathrm{OOCH}\right)\left(\mathrm{PMe}_{3}\right)(7)$

S11

Crystallographic parameters for 1, 2-PMe $3,2-\mathbf{P P h}_{3}, \mathbf{5 , 6 a}$

S11-12 


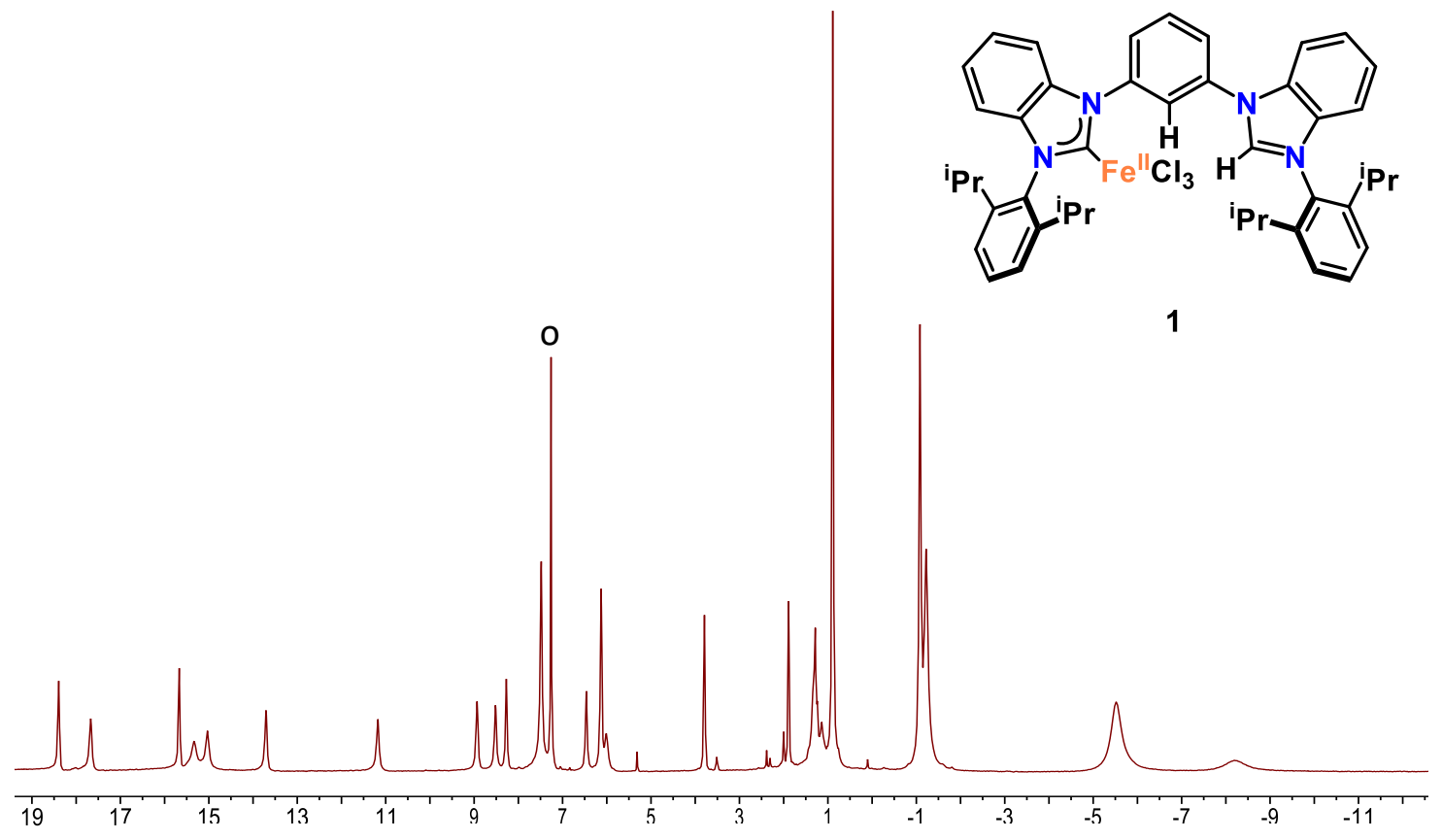

Figure S1. $\mathrm{H}_{2}\left({ }^{\mathrm{DIPP}} \mathrm{CCC}\right) \mathrm{FeCl}_{3}(\mathbf{1}){ }^{1} \mathrm{H} \mathrm{NMR}$ spectrum $\left({ }^{\circ}\right.$ denotes $\left.\mathrm{CDCl}_{3}\right)$.

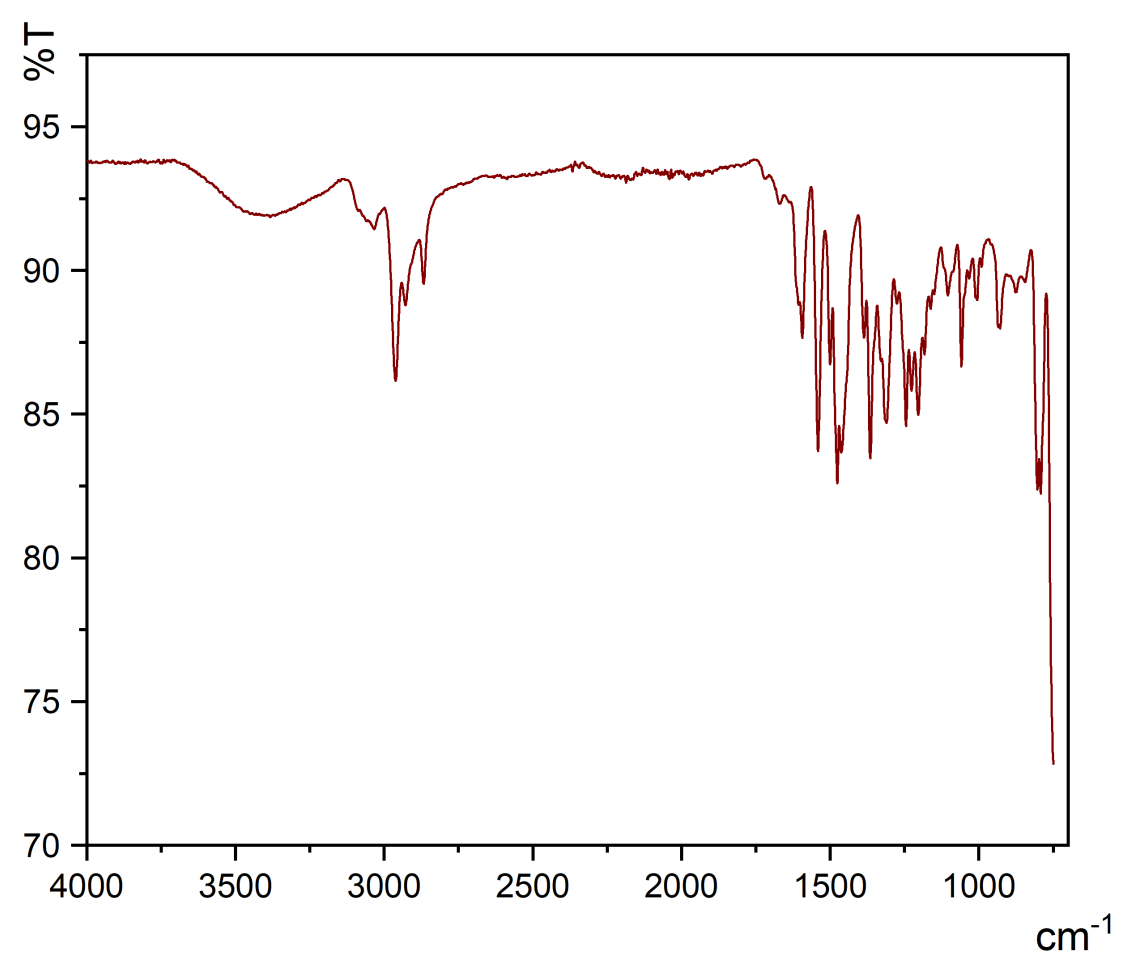

Figure S2. $\mathrm{H}_{2}\left({ }^{\mathrm{DIPP}} \mathrm{CCC}\right) \mathrm{FeCl}_{3}$ (1) IR spectrum. 


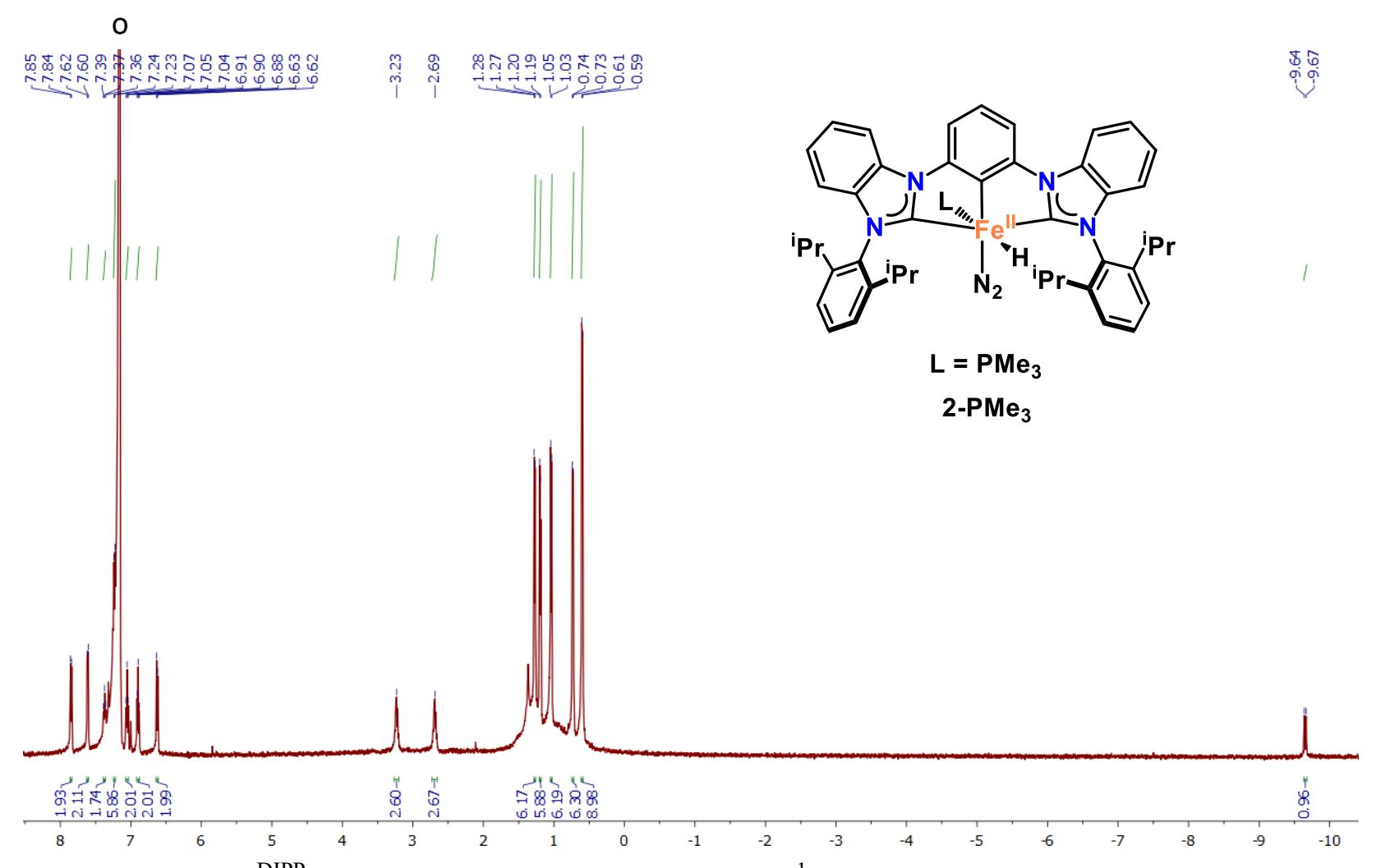

Figure S3. $\mathrm{H}\left({ }^{\mathrm{DIPP}} \mathrm{CCC}\right) \mathrm{Fe}(\mathrm{II}) \mathrm{H}\left(\mathrm{PMe}_{3}\right)\left(\mathrm{N}_{2}\right)\left(\mathbf{2}-\mathbf{P M e}_{3}\right){ }^{1} \mathrm{H} \mathrm{NMR}$ spectrum $\left({ }^{\mathrm{o}}\right.$ denotes $\left.\mathrm{C}_{6} \mathrm{D}_{6}\right)$.

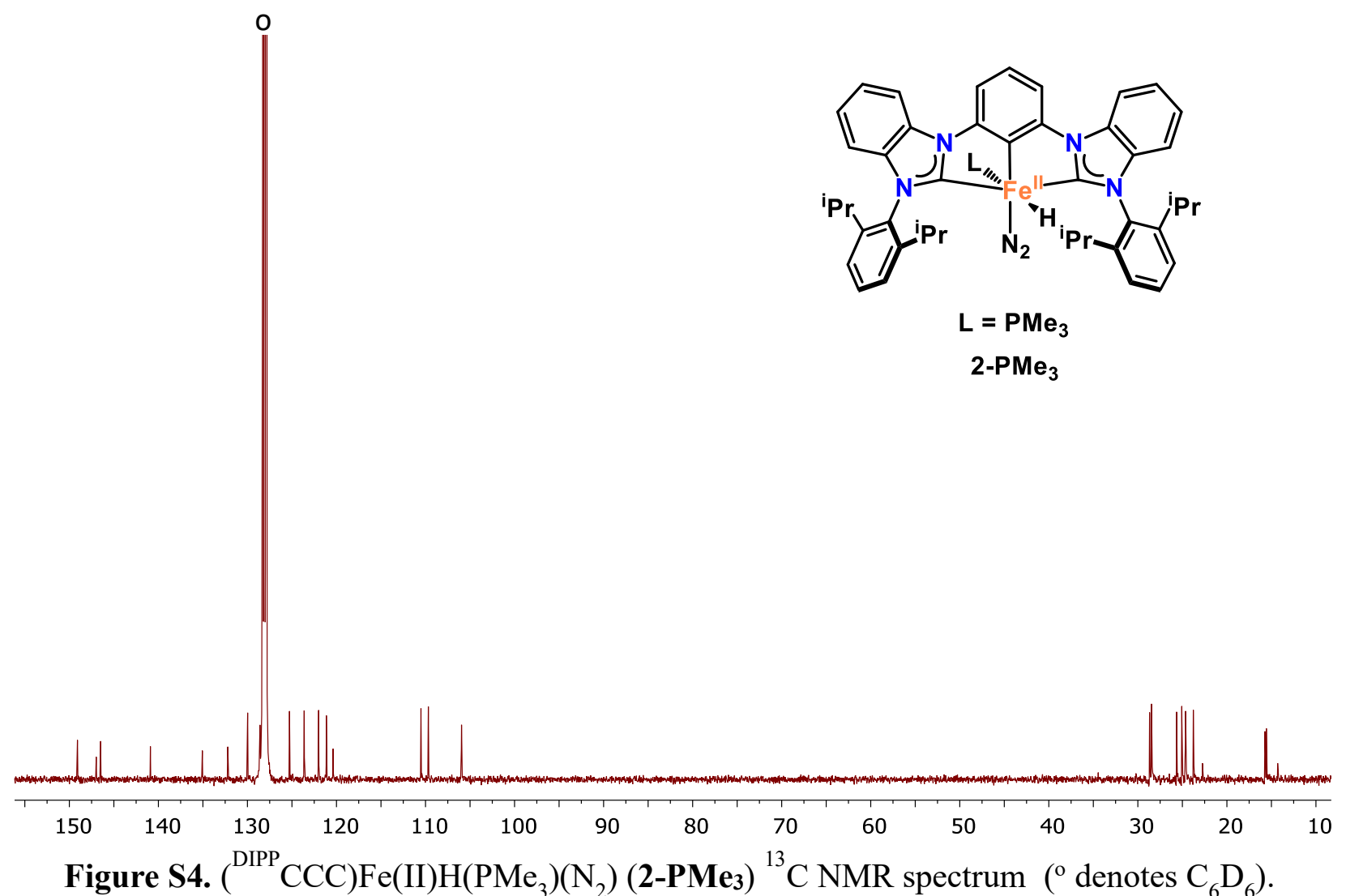




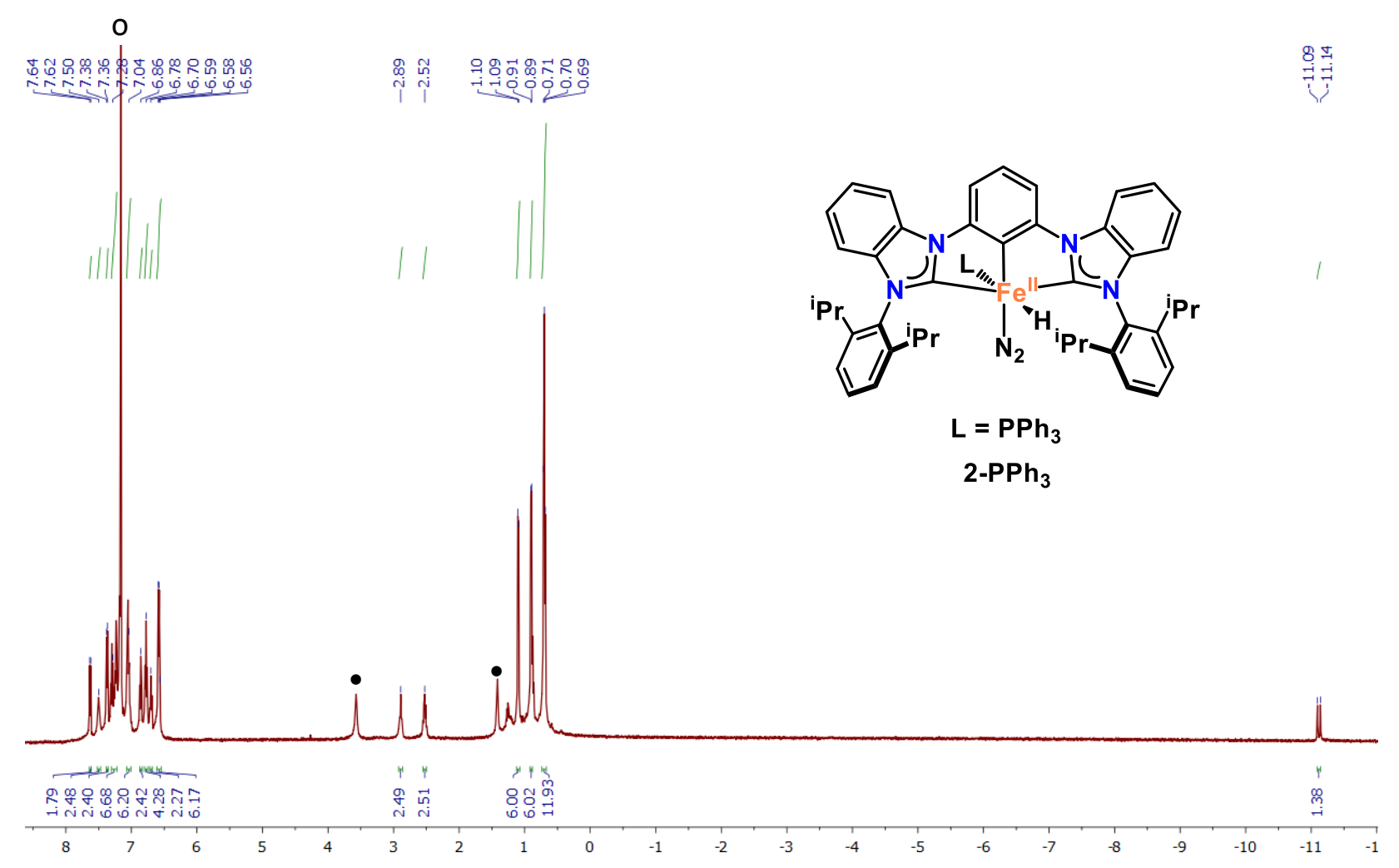

Figure S5. $\left({ }^{\mathrm{DIPP}} \mathrm{CCC}\right) \mathrm{Fe}(\mathrm{II}) \mathrm{H}\left(\mathrm{PPh}_{3}\right)\left(\mathrm{N}_{2}\right)\left(\mathbf{2}-\mathrm{PPh}_{3}\right){ }^{1} \mathrm{H}$ NMR spectrum $(\bullet$ denotes THF, ${ }^{\circ}$ denotes $\mathrm{C}_{6} \mathrm{D}_{6}$ ).

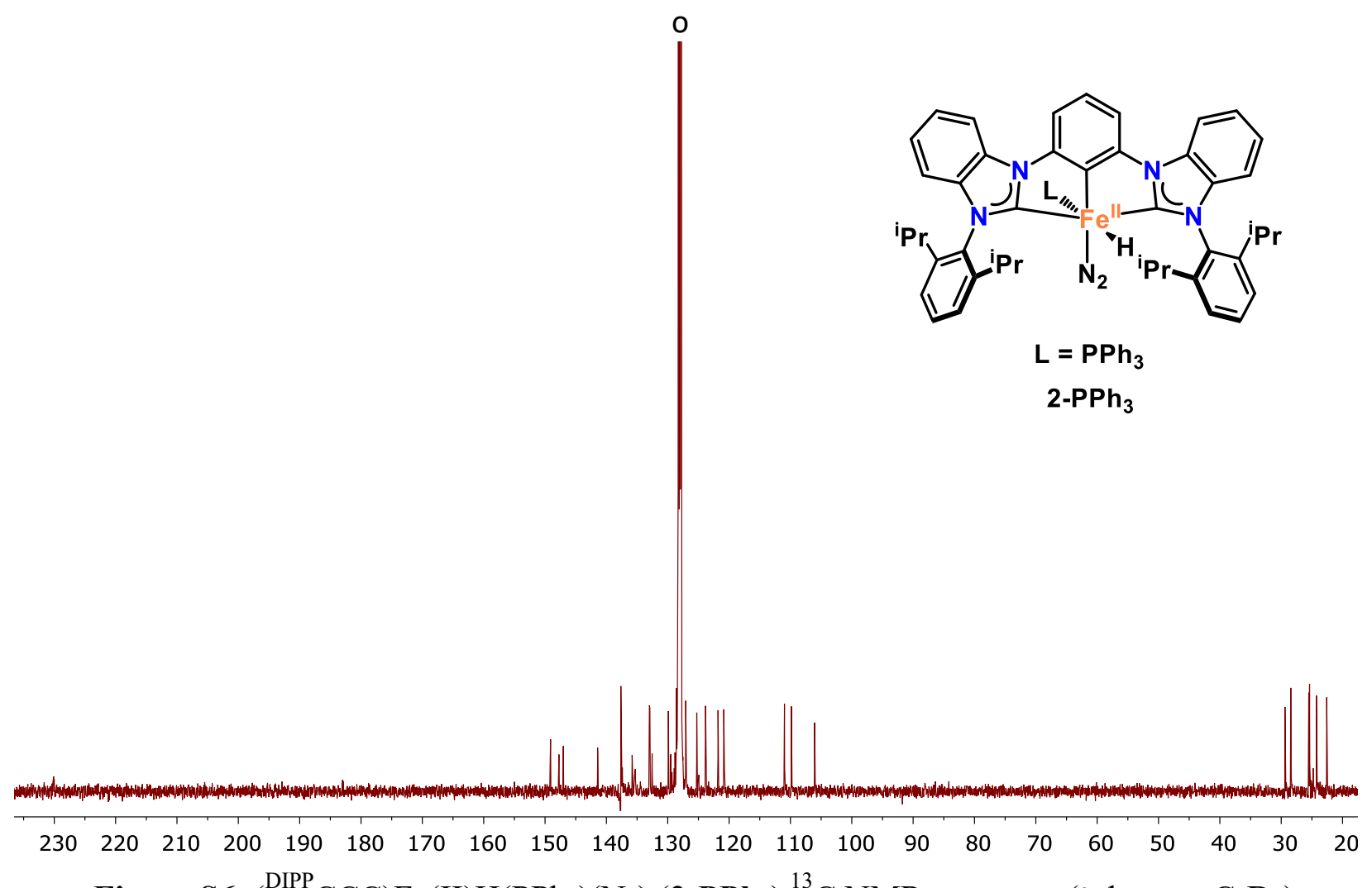

Figure S6. $\left({ }^{\mathrm{DIPP}} \mathrm{CCC}\right) \mathrm{Fe}(\mathrm{II}) \mathrm{H}\left(\mathrm{PPh}_{3}\right)\left(\mathrm{N}_{2}\right)\left(\mathbf{2}-\mathbf{P P h}_{3}\right){ }^{13} \mathrm{C}$ NMR spectrum $\left({ }^{\circ}\right.$ denotes $\left.\mathrm{C}_{6} \mathrm{D}_{6}\right)$. 


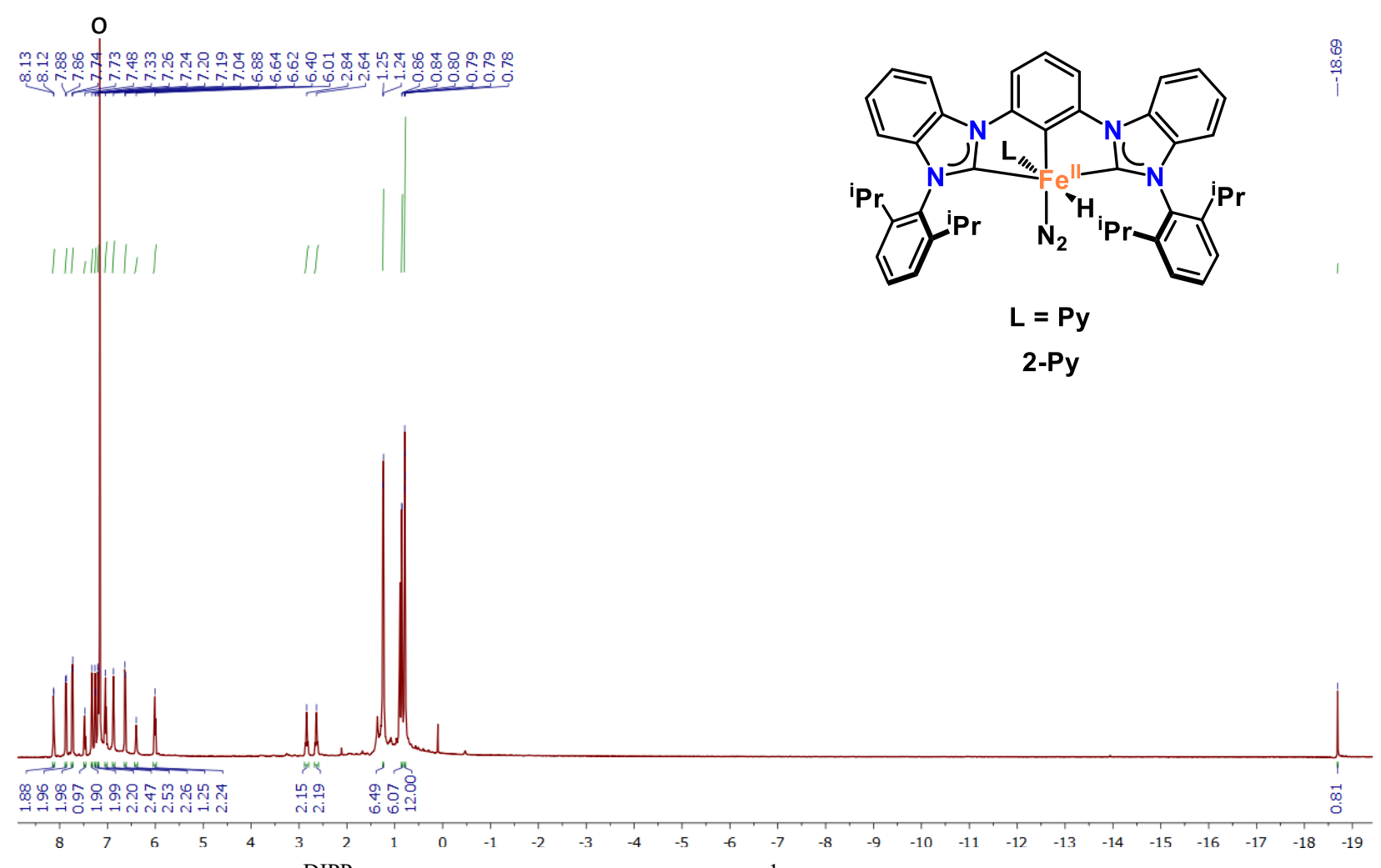

Figure S7. $\left({ }^{\mathrm{DIPP}} \mathrm{CCC}\right) \mathrm{Fe}(\mathrm{II}) \mathrm{H}(\mathrm{Py})\left(\mathrm{N}_{2}\right)(\mathbf{2}-\mathrm{Py}){ }^{1} \mathrm{H}$ NMR spectrum $\left({ }^{\circ}\right.$ denotes $\left.\mathrm{C}_{6} \mathrm{D}_{6}\right)$.

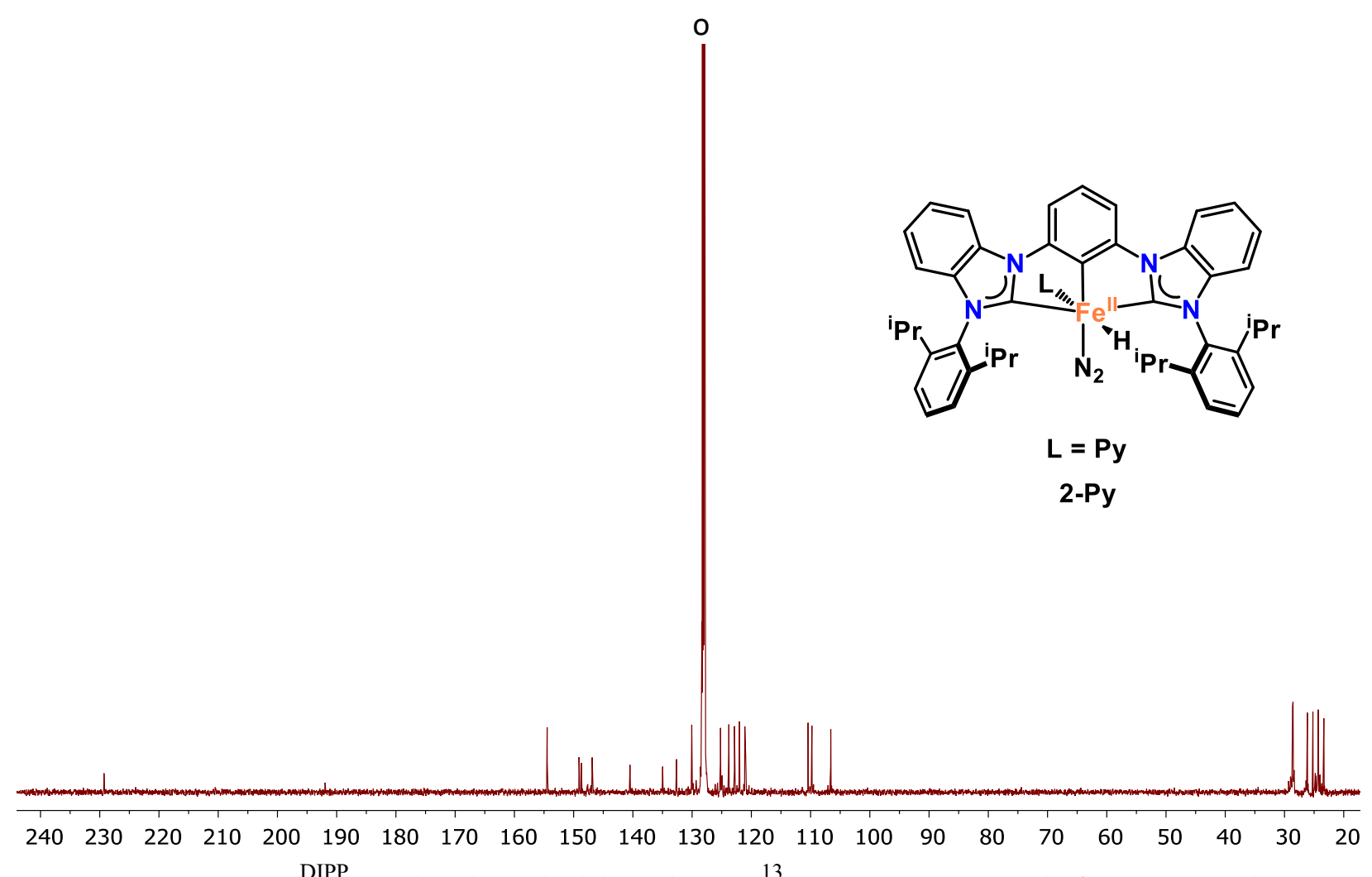

Figure S8. $\left({ }^{\mathrm{DIPP}} \mathrm{CCC}\right) \mathrm{Fe}(\mathrm{II}) \mathrm{H}(\mathrm{Py})\left(\mathrm{N}_{2}\right)(\mathbf{2}-\mathrm{Py}){ }^{13} \mathrm{C}$ NMR spectrum $\left({ }^{\mathrm{o}}\right.$ denotes $\left.\mathrm{C}_{6} \mathrm{D}_{6}\right)$. 
0
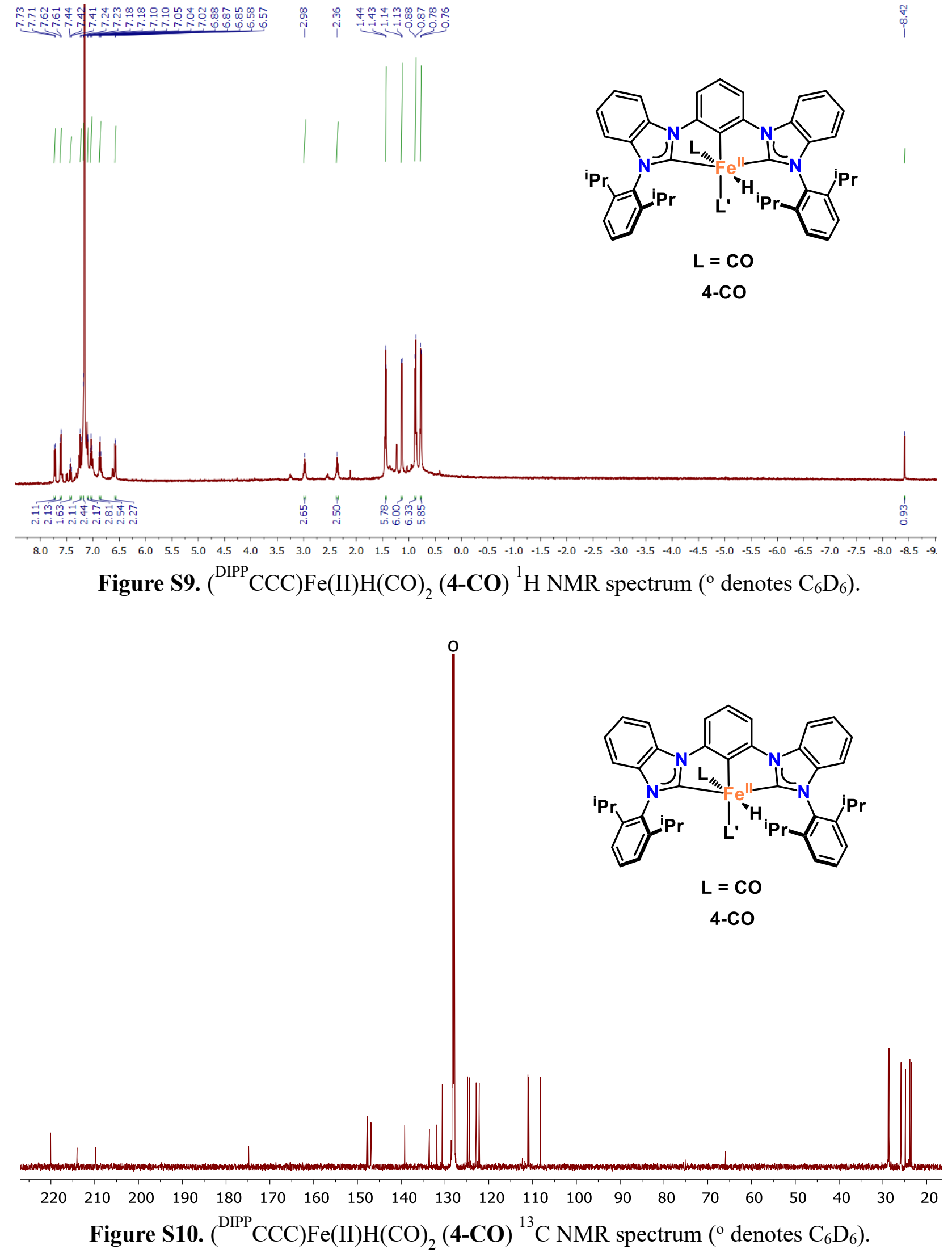


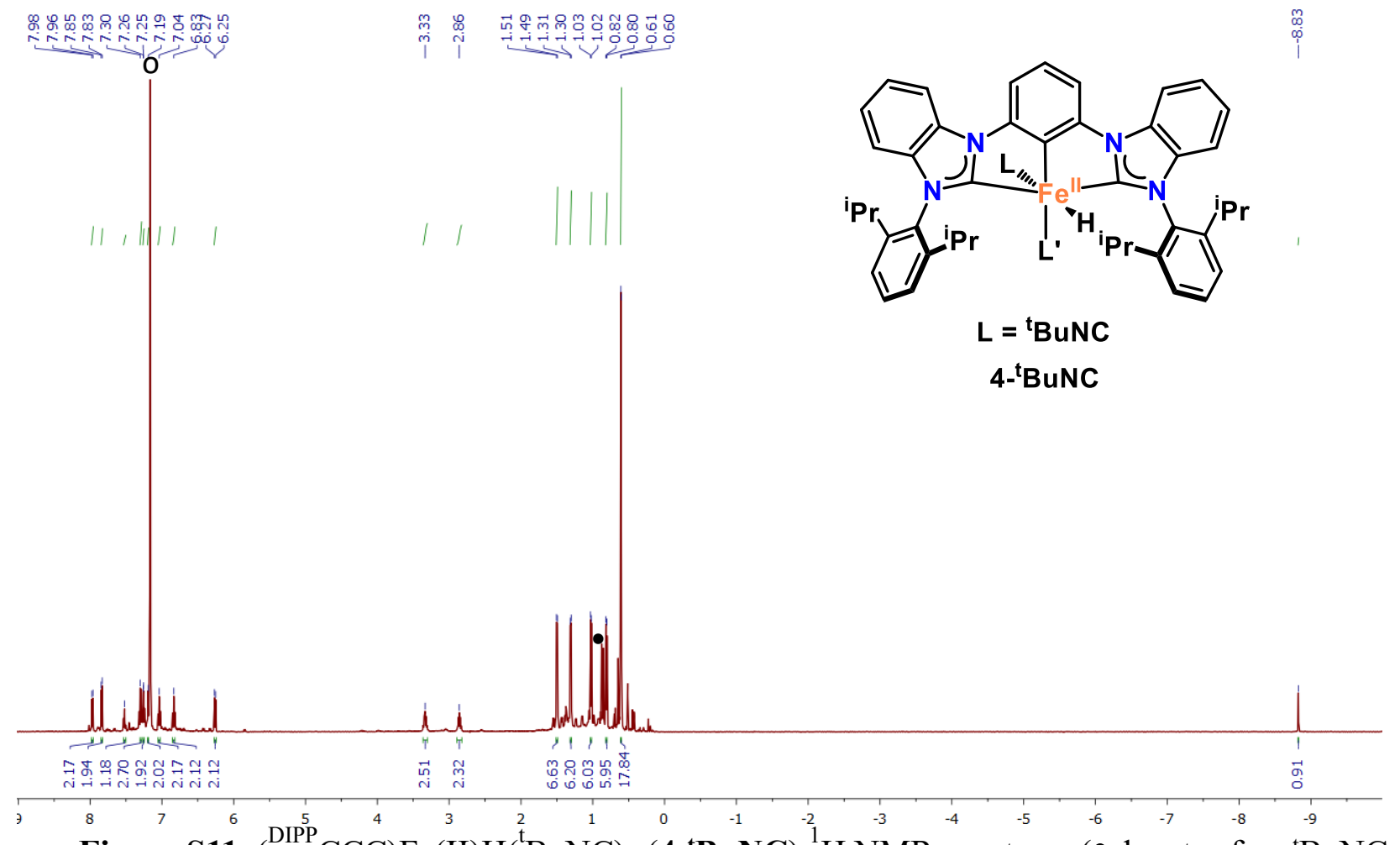

Figure S11. $\left({ }^{\mathrm{DIPP}} \mathrm{CCC}\right) \mathrm{Fe}(\mathrm{II}) \mathrm{H}\left({ }^{\mathrm{t}} \mathrm{BuNC}\right)_{2}\left(\mathbf{4}^{\mathrm{t}} \mathrm{BuNC}\right){ }^{1} \mathrm{H}$ NMR spectrum $\left(\bullet\right.$ denotes free ${ }^{\mathrm{t}} \mathrm{BuNC}$ ${ }^{o}$ denotes $\left.\mathrm{C}_{6} \mathrm{D}_{6}\right)$.

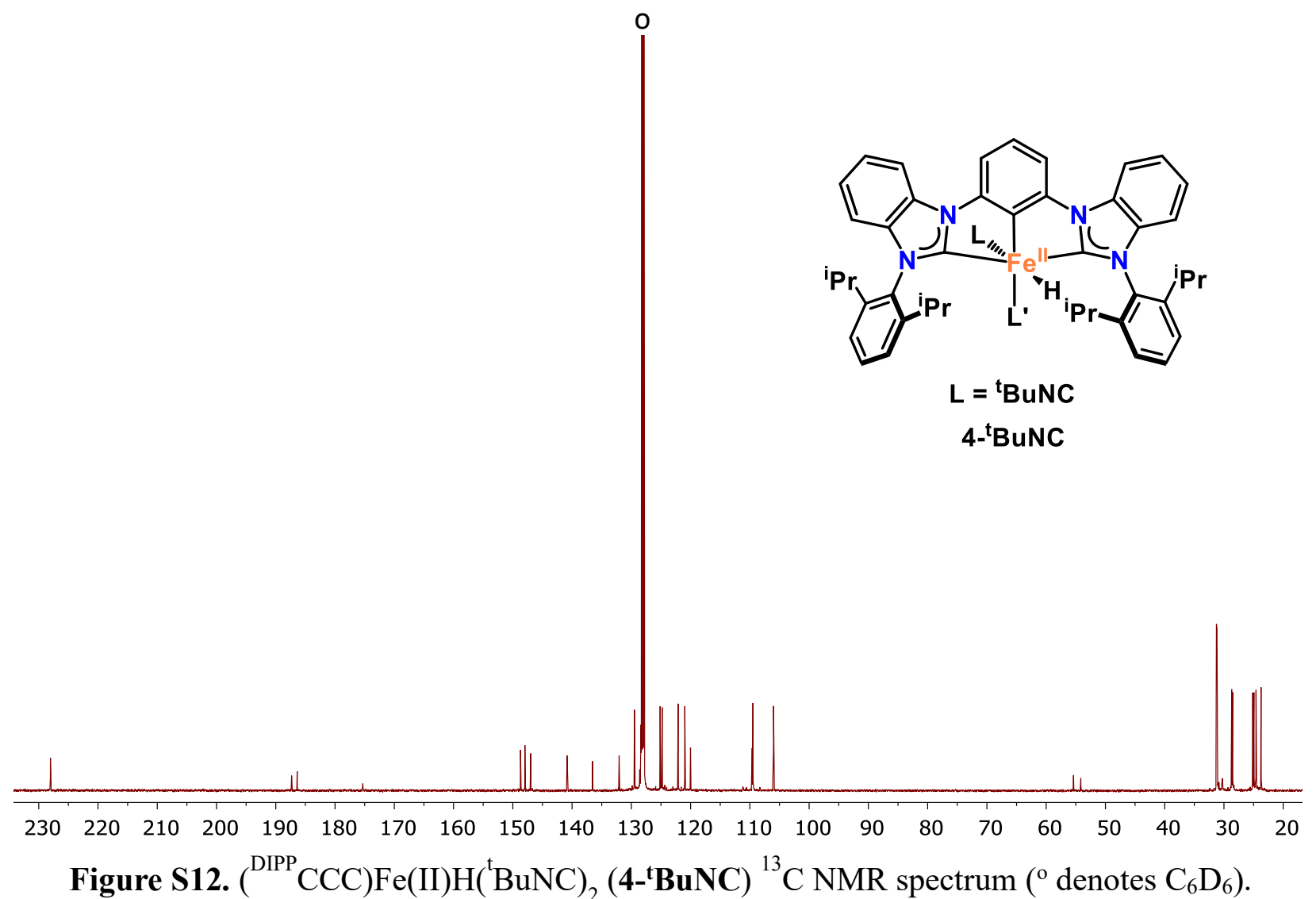




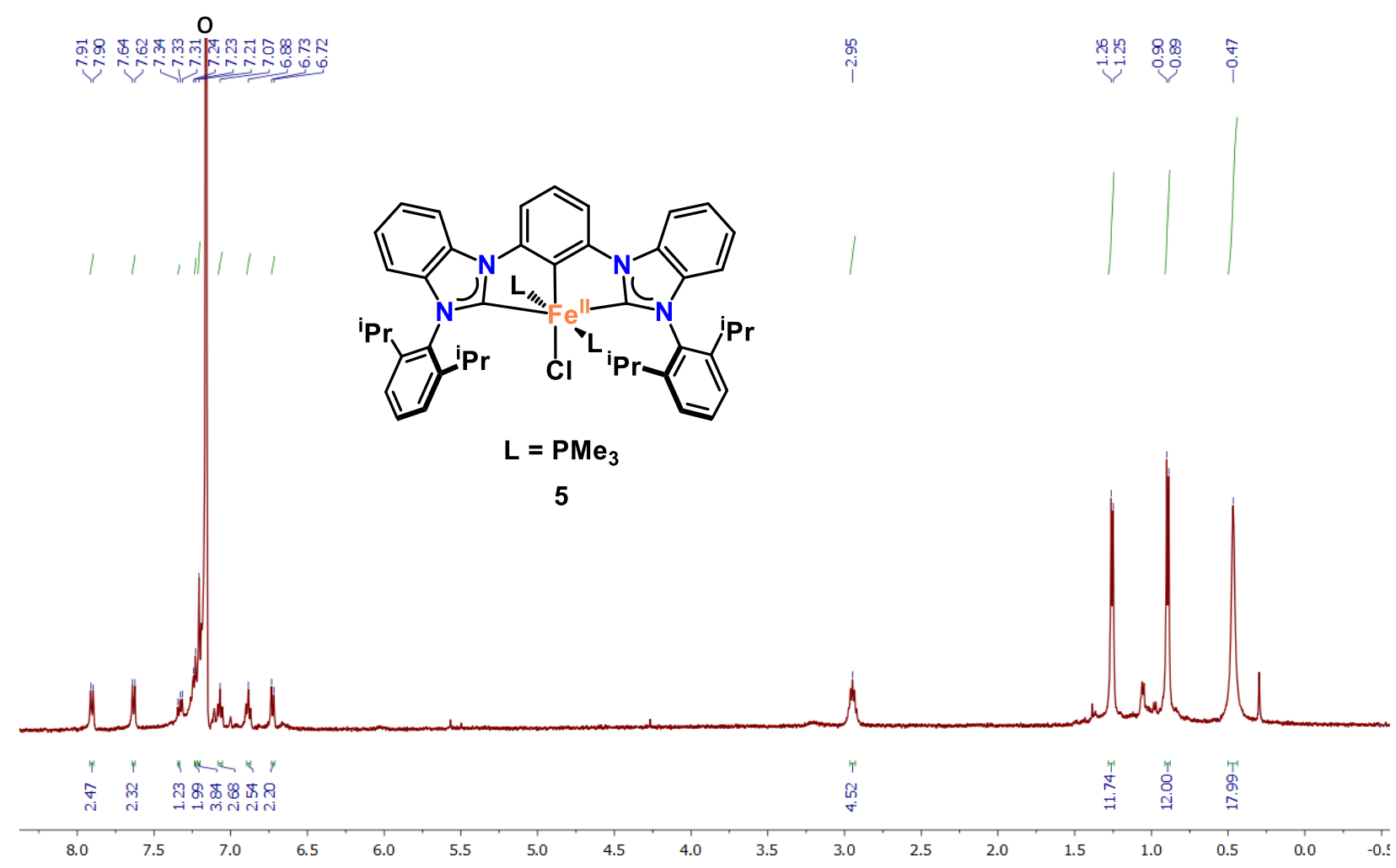

Figure S13. $\left({ }^{\mathrm{DIPP}} \mathrm{CCC}\right) \mathrm{Fe}(\mathrm{II}) \mathrm{Cl}\left(\mathrm{PMe}_{3}\right)_{2}(\mathbf{5}){ }^{1} \mathrm{H}$ NMR spectrum $\left({ }^{\circ}\right.$ denotes $\left.\mathrm{C}_{6} \mathrm{D}_{6}\right)$.

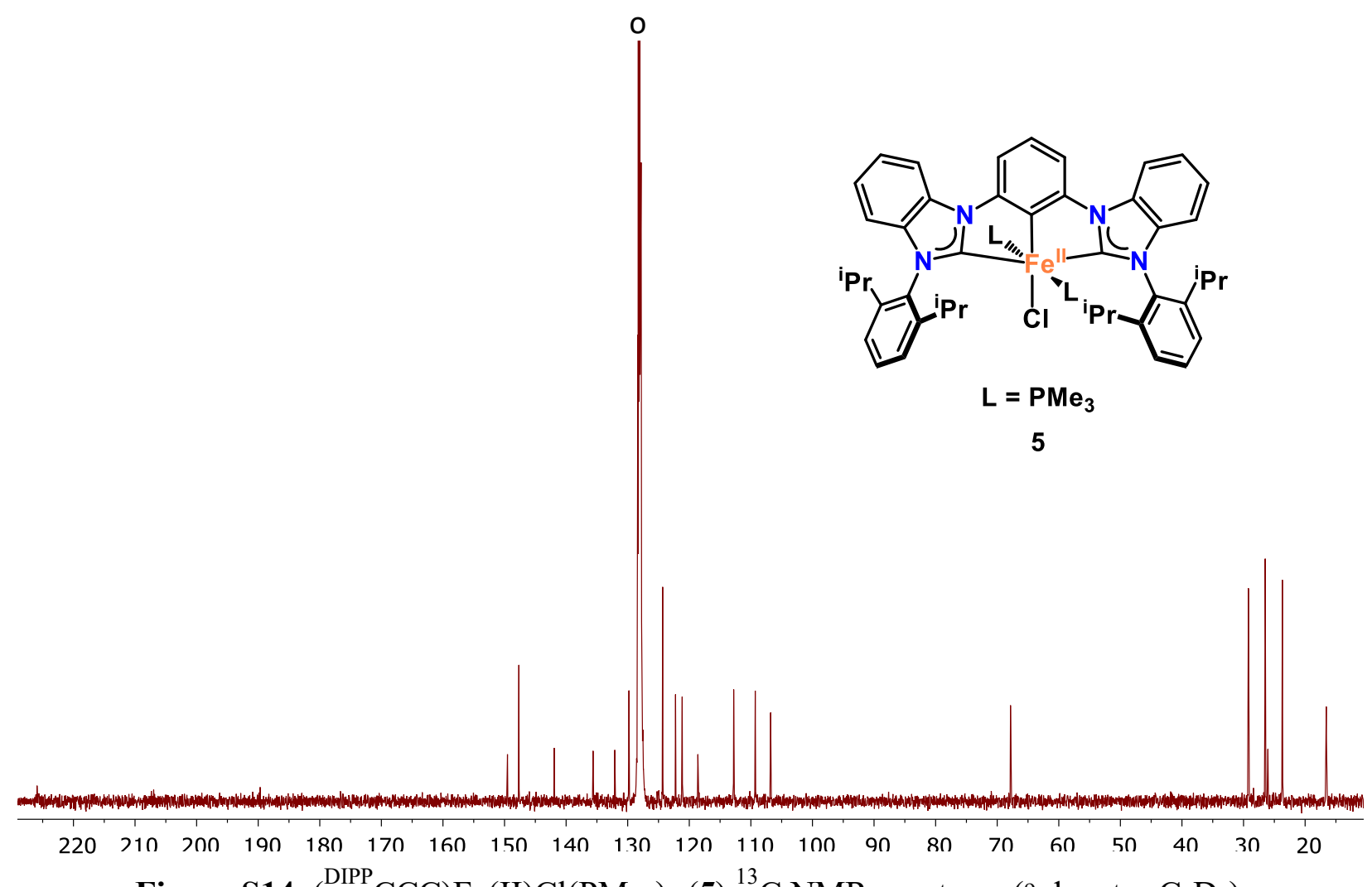

Figure S14. $\left({ }^{\mathrm{DIPP}} \mathrm{CCC}\right) \mathrm{Fe}(\mathrm{II}) \mathrm{Cl}\left(\mathrm{PMe}_{3}\right)_{2}(\mathbf{5}){ }^{13} \mathrm{C}$ NMR spectrum $\left({ }^{\circ}\right.$ denotes $\left.\mathrm{C}_{6} \mathrm{D}_{6}\right)$. 


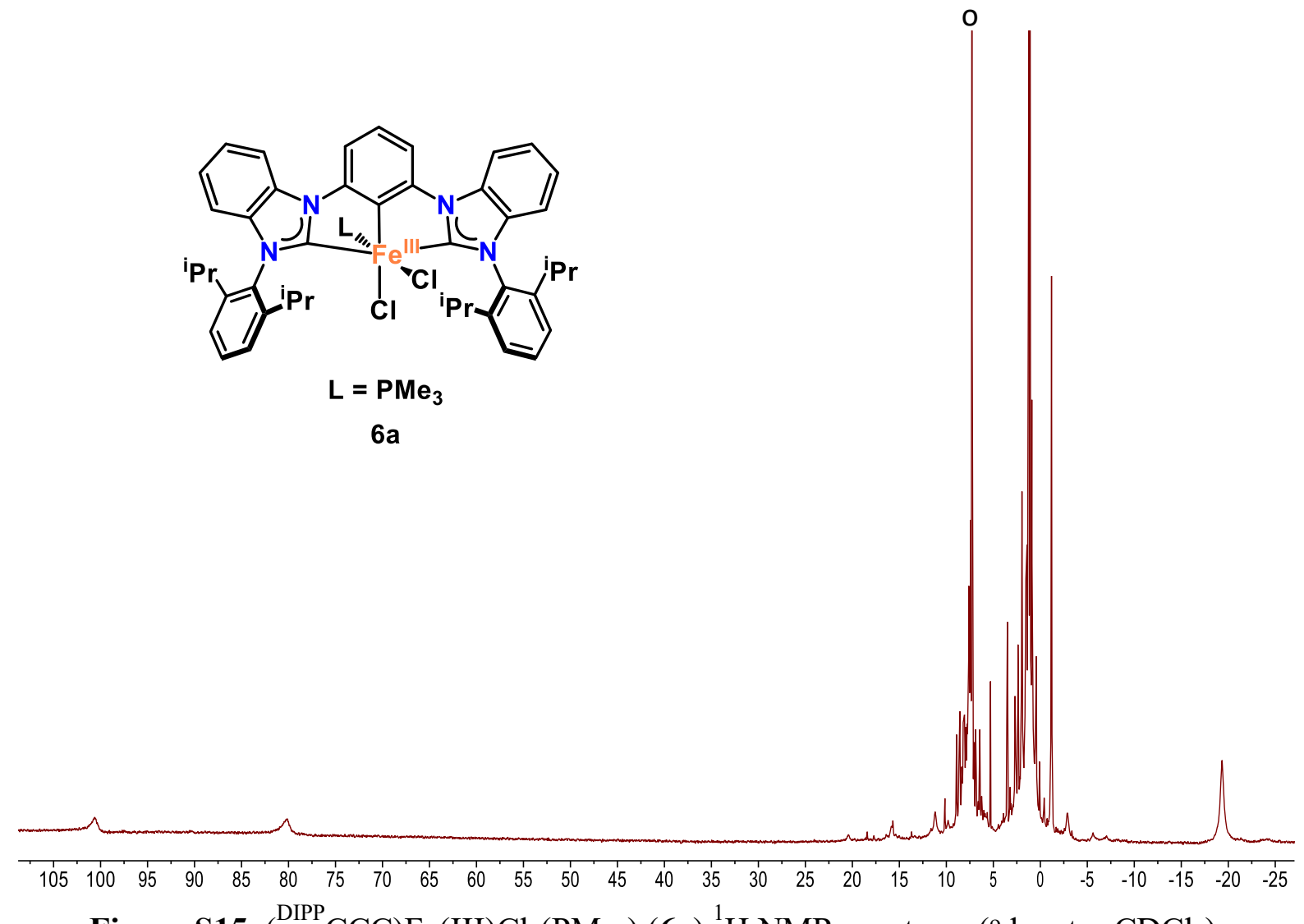

Figure S15. ( $\left.{ }^{\mathrm{DIPP}} \mathrm{CCC}\right) \mathrm{Fe}(\mathrm{III}) \mathrm{Cl}_{3}\left(\mathrm{PMe}_{3}\right)(\mathbf{6 a}){ }^{1} \mathrm{H}$ NMR spectrum $\left({ }^{\circ}\right.$ denotes $\left.\mathrm{CDCl}_{3}\right)$.

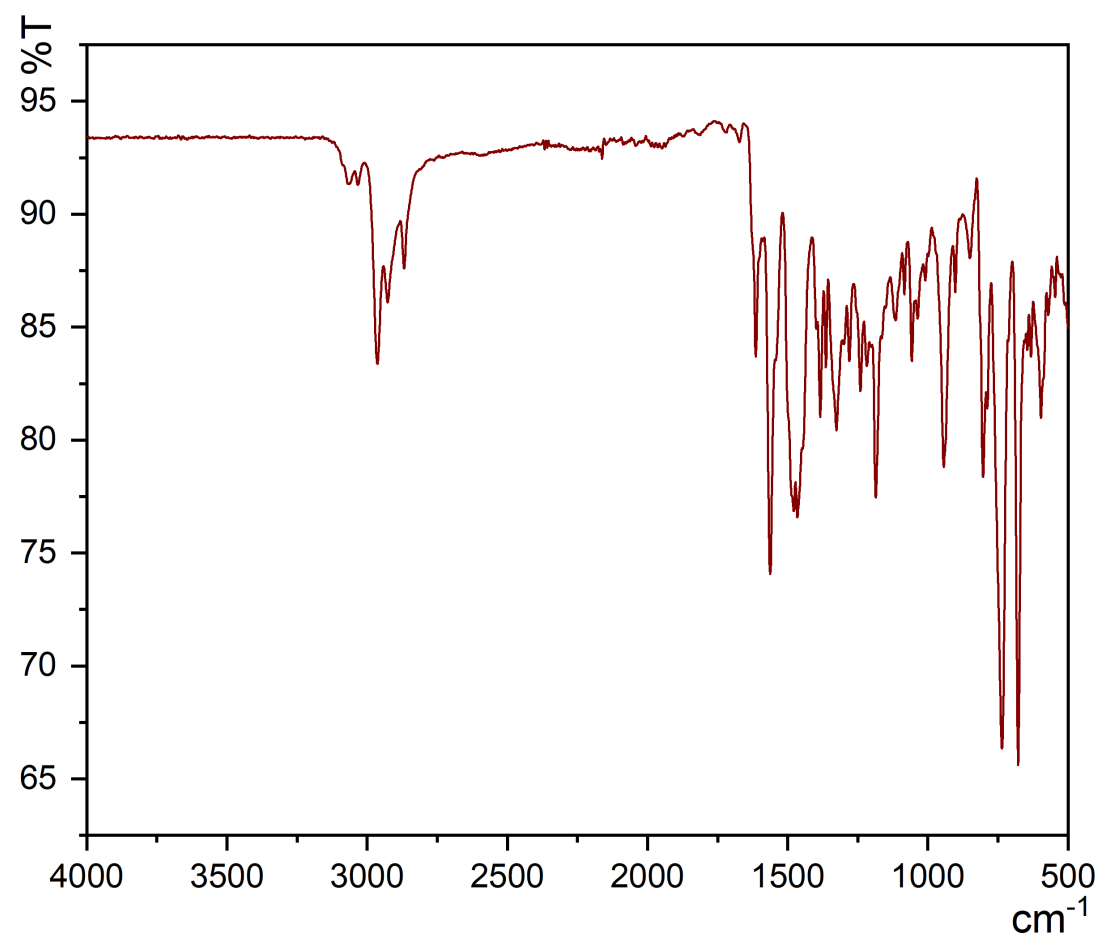

Figure S16. $\left({ }^{\mathrm{DIPP}} \mathrm{CCC}\right) \mathrm{Fe}(\mathrm{III}) \mathrm{Cl}_{2}\left(\mathrm{PMe}_{3}\right)(\mathbf{6 a})$ IR spectrum. 


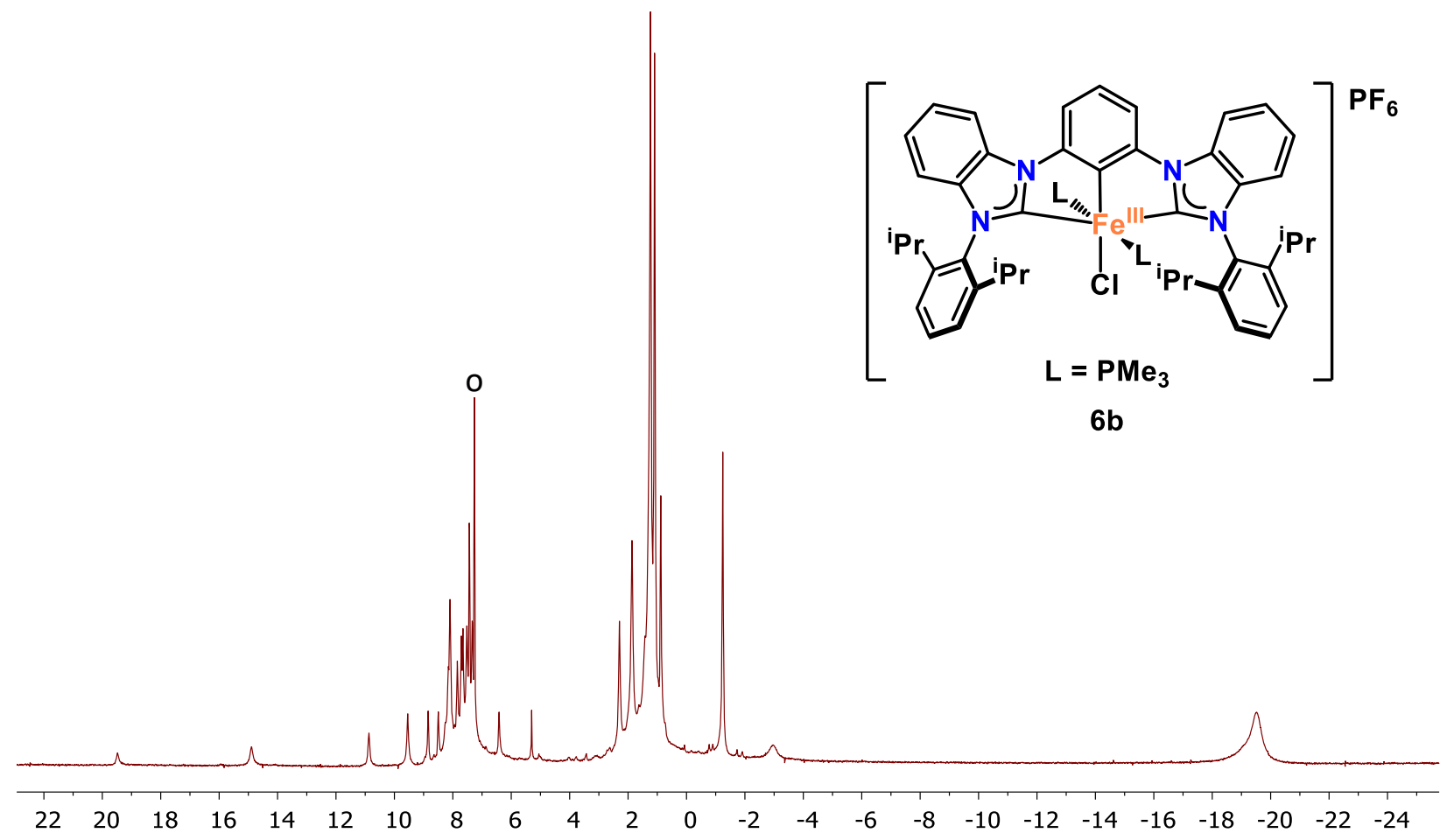

Figure S17. $\left({ }^{\mathrm{DIPP}} \mathrm{CCC}\right) \mathrm{Fe}(\mathrm{III}) \mathrm{Cl}\left(\mathrm{PMe}_{3}\right)_{2} \mathrm{PF}_{6}(\mathbf{6 b}){ }^{1} \mathrm{H}$ NMR spectrum $\left({ }^{\circ}\right.$ denotes $\left.\mathrm{C}_{6} \mathrm{D}_{6}\right)$.

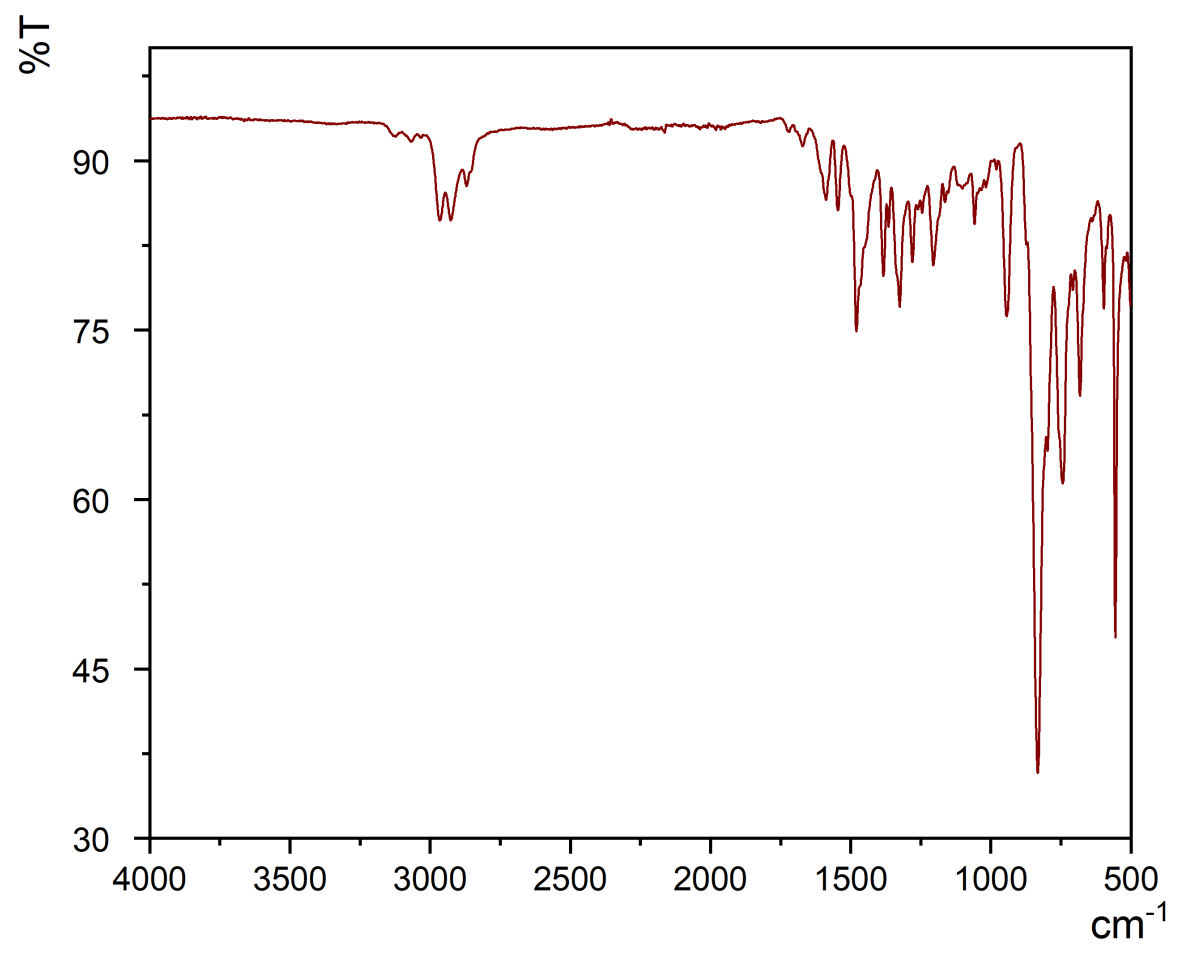

Figure S18. $\left({ }^{\mathrm{DIPP}} \mathrm{CCC}\right) \mathrm{Fe}(\mathrm{III}) \mathrm{Cl}\left(\mathrm{PMe}_{3}\right)_{2} \mathrm{PF}_{6}(\mathbf{6 b}) \mathrm{IR}$ spectrum. 


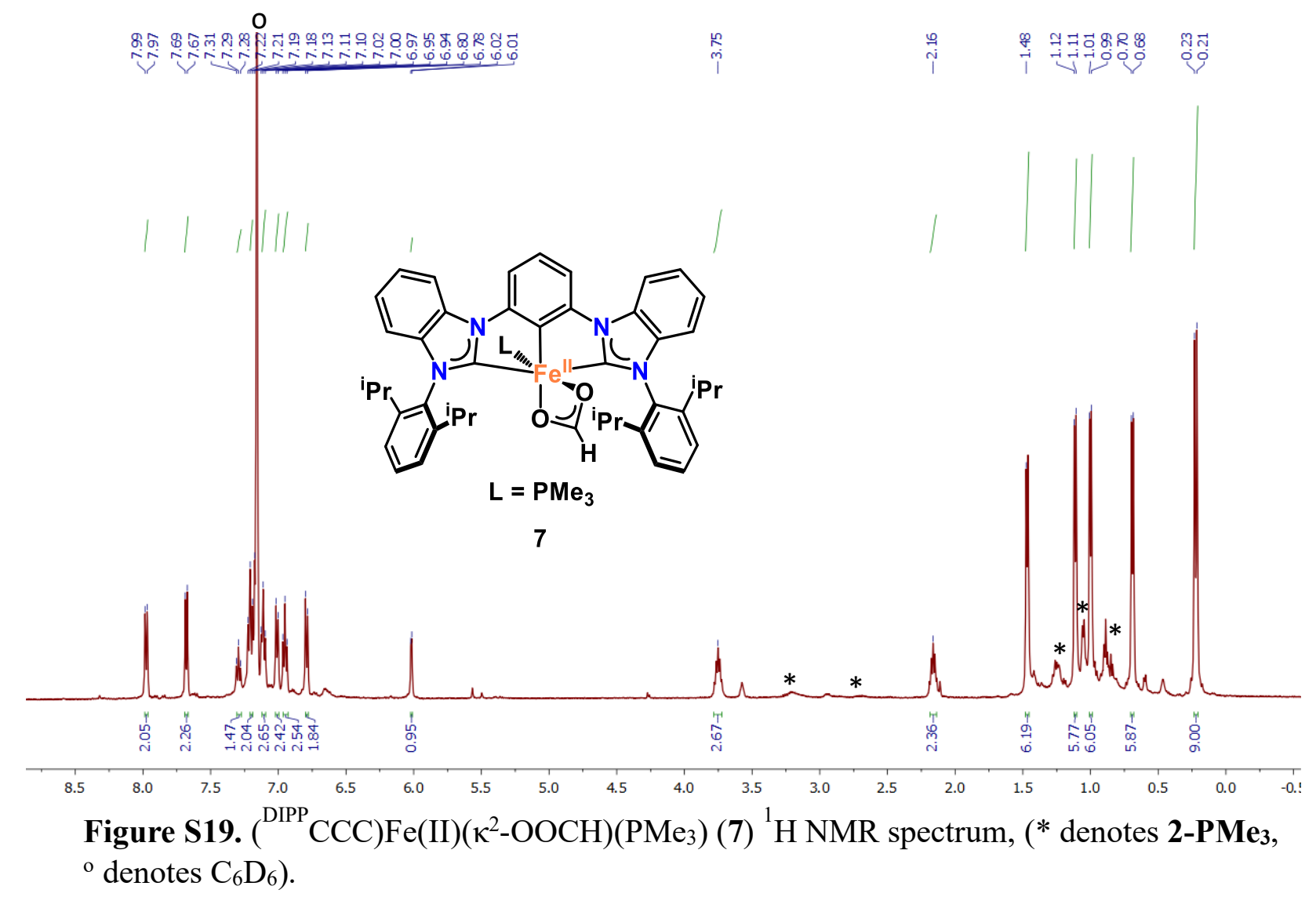

Table S1. Crystallographic parameters for $\mathrm{H}_{2}\left({ }^{\mathrm{DIPP}} \mathrm{CCC}\right) \mathrm{Fe}(\mathrm{II}) \mathrm{Cl}_{3}$ and $\left({ }^{\text {DIPP }} \mathrm{CCC}\right) \mathrm{Fe}(\mathrm{II}) \mathrm{H}\left(\mathrm{PMe}_{3}\right)\left(\mathrm{N}_{2}\right)$.

\begin{tabular}{|c|c|c|}
\hline Compound & $\mathrm{H}_{2}\left({ }^{\mathrm{DIPP}} \mathrm{CCC}\right) \mathrm{Fe}(\mathrm{II}) \mathrm{Cl}_{3}(\mathbf{1})$ & $\begin{array}{c}\left({ }^{\mathrm{DIPP}} \mathrm{CCC}\right) \mathrm{Fe}(\mathrm{II}) \mathrm{H}\left(\mathrm{PMe}_{3}\right)\left(\mathrm{N}_{2}\right) \\
(\mathbf{2}-\mathbf{P M e})\end{array}$ \\
\hline Empirical formula & $\mathrm{C}_{44} \mathrm{H}_{47} \mathrm{~N}_{4} \mathrm{Cl}_{3} \mathrm{Fe}$ & $\mathrm{C}_{52} \mathrm{H}_{67} \mathrm{~N}_{6} \mathrm{PFe}$ \\
\hline Formula weight & 794.05 & 862.94 \\
\hline Temperature/K & $100(2)$ & $100(2)$ \\
\hline Crystal system & monoclinic & triclinic \\
\hline Space group & $\mathrm{P} 2{ }_{1} / \mathrm{n}$ & $\mathrm{P}-1$ \\
\hline $\mathrm{a} / \AA$ & $16.2332(7)$ & $12.0449(5)$ \\
\hline $\mathrm{b} / \AA$ & $17.6703(7)$ & $14.6183(6)$ \\
\hline $\mathrm{c} / \AA$ & $16.3741(7)$ & $15.7848(7)$ \\
\hline$\alpha /^{\circ}$ & 90.00 & $107.2907(18)$ \\
\hline$\beta /{ }^{\circ}$ & $98.5058(15)$ & $108.0980(19)$ \\
\hline$\gamma /{ }^{\circ}$ & 90.00 & $103.6701(19)$ \\
\hline Volume $/ \AA^{3}$ & $4645.2(3)$ & $2351.23(18)$ \\
\hline $\mathrm{Z}$ & 4 & 2 \\
\hline Reflections collected & 43720 & 82885 \\
\hline Independent reflections & $\begin{array}{c}8519\left[\mathrm{R}_{\text {int }}=0.0289\right. \\
\left.\mathrm{R}_{\text {sigma }}=0.0205\right]\end{array}$ & $\begin{array}{c}17197\left[\mathrm{R}_{\text {int }}=0.0426\right. \\
\left.\mathrm{R}_{\text {sigma }}=0.0366\right]\end{array}$ \\
\hline Goodness-of-fit & 1.058 & 1.022 \\
\hline Final $\mathrm{R}$ indexes $[\mathrm{I}>=2 \sigma(\mathrm{I})]$ & $\mathrm{R}_{1}=0.0338, \mathrm{wR}_{2}=0.0855$ & $\mathrm{R}_{1}=0.0544, \mathrm{wR}_{2}=0.0934$ \\
\hline Final $\mathrm{R}$ indexes [all data] & $\mathrm{R}_{1}=0.0423, \mathrm{w} \mathrm{R}_{2}=0.0907$ & $\mathrm{R}_{1}=0.0388, \mathrm{wR}_{2}=0.1021$ \\
\hline
\end{tabular}


Table S2. Crystallographic parameters $\left({ }^{\mathrm{DIPP}} \mathrm{CCC}\right) \mathrm{Fe}(\mathrm{II}) \mathrm{Cl}\left(\mathrm{PMe}_{3}\right)_{2}$ and $\left({ }^{\text {DIPP }} \mathrm{CCC}\right) \mathrm{Fe}(\mathrm{III})(\mathrm{Cl})_{2}\left(\mathrm{PMe}_{3}\right)$.

\begin{tabular}{|c|c|c|}
\hline Compound & $\begin{array}{c}\left({ }^{(\mathrm{DIPP}} \mathrm{CCC}\right) \mathrm{Fe}(\mathrm{II}) \mathrm{H}\left(\mathrm{PPh}_{3}\right)\left(\mathrm{N}_{2}\right) \\
\left(\mathbf{2}-\mathbf{P P h}_{3}\right)\end{array}$ & $\left({ }^{\mathrm{DIPP}} \mathrm{CCC}\right) \mathrm{Fe}(\mathrm{II}) \mathrm{Cl}\left(\mathrm{PMe}_{3}\right)_{2}(\mathbf{5})$ \\
\hline Empirical formula & $\mathrm{C}_{62} \mathrm{H}_{61} \mathrm{~N}_{6} \mathrm{PFe}$ & $\mathrm{C}_{50} \mathrm{H}_{63} \mathrm{~N}_{4} \mathrm{P}_{2} \mathrm{ClFe}$ \\
\hline Formula weight & 976.98 & 873.28 \\
\hline Temperature/K & $100(2)$ & $100(2)$ \\
\hline Crystal system & triclinic & monoclinic \\
\hline Space group & P-1 & $\mathrm{C} 2 / \mathrm{c}$ \\
\hline $\mathrm{a} / \AA \AA$ & $11.3052(6)$ & $21.1714(11)$ \\
\hline $\mathrm{b} / \AA$ & $17.0256(9)$ & $11.0332(5)$ \\
\hline $\mathrm{c} / \AA \AA$ & $18.5371(10)$ & $18.8162(10)$ \\
\hline$\alpha /^{\circ}$ & $67.3098(10)$ & 90 \\
\hline$\beta /{ }^{\circ}$ & $77.1910(9)$ & $90.101(2)$ \\
\hline$\gamma /{ }^{\circ}$ & $73.9916(9)$ & 90 \\
\hline Volume $/ \AA^{3}$ & $3137.3(3)$ & $4395.2(4)$ \\
\hline $\mathrm{Z}$ & 2 & 4 \\
\hline Reflections collected & 11080 & 92760 \\
\hline Independent reflections & $\begin{array}{c}4062\left[R_{\text {int }}=0.0467,\right. \\
\left.R_{\text {sigma }}=0.0396\right]\end{array}$ & $\begin{array}{c}4062\left[R_{\text {int }}=0.0472,\right. \\
\left.R_{\text {sigma }}=0.0135\right]\end{array}$ \\
\hline Goodness-of-fit & 0.885 & 1.102 \\
\hline Final R indexes $[\mathrm{I}>=2 \sigma(\mathrm{I})]$ & $\mathrm{R}_{1}=0.0396, \mathrm{wR}_{2}=0.1263$ & $\mathrm{R}_{1}=0.0479, \mathrm{wR}_{2}=0.1181$ \\
\hline Final R indexes [all data] & $\mathrm{R}_{1}=0.0467, \mathrm{wR}_{2}=0.1329$ & $\mathrm{R}_{1}=0.0510, \mathrm{wR}_{2}=0.1201$ \\
\hline
\end{tabular}

Table S3. Crystallographic parameters for $\left({ }^{\mathrm{DIPP}} \mathrm{CCC}\right) \mathrm{Fe}(\mathrm{III})(\mathrm{Cl})_{2}\left(\mathrm{PMe}_{3}\right)$.

\begin{tabular}{|c|c|}
\hline Compound & $\begin{array}{c}\left({ }^{\text {DIPPCCC }}\right) \mathrm{Fe}(\mathrm{III})(\mathrm{Cl})_{2}\left(\mathrm{PMe}_{3}\right) \\
(\mathbf{6 a})\end{array}$ \\
\hline Empirical formula & $\mathrm{C}_{47} \mathrm{H}_{54} \mathrm{Cl}_{2} \mathrm{FeN}_{4} \mathrm{P}$ \\
\hline Formula weight & 832.66 \\
\hline Temperature/K & $112(2)$ \\
\hline Crystal system & triclinic \\
\hline Space group & $\mathrm{P}-1$ \\
\hline $\mathrm{a} / \AA$ & $10.7730(17)$ \\
\hline $\mathrm{b} / \AA$ & $14.849(2)$ \\
\hline $\mathrm{c} / \AA$ & $16.687(2)$ \\
\hline$\alpha /{ }^{\circ}$ & $91.381(8)$ \\
\hline$\beta /{ }^{\circ}$ & $95.761(8)$ \\
\hline$\gamma^{\circ}$ & $93.318(8)$ \\
\hline Volume/ $/ \AA^{3}$ & $2650.2(7)$ \\
\hline$Z$ & 2 \\
\hline Reflections collected & 18868 \\
\hline Independent reflections & $18868\left[\mathrm{R}_{\text {sigma }}=0.1108\right]$ \\
\hline Goodness-of-fit & 1.080 \\
\hline Final R indexes $[\mathrm{I}>=2 \sigma(\mathrm{I})]$ & $\mathrm{R}_{1}=0.0867, \mathrm{wR}_{2}=0.1941$ \\
\hline Final R indexes [all data] & $\mathrm{R}_{1}=0.1373, \mathrm{wR}_{2}=0.2223$ \\
\hline
\end{tabular}

